\title{
Dynamical principles in neuronal systems and robotics
}

Published online: 6 December 2006

(C) Springer-Verlag 2006

A dream that has existed for centuries is to build a machine able to replicate human functions. Some impressive working robots exist today that are in some cases able to replace humans in dangerous environments, assist with factory assembly tasks, and even provide companionship and entertainment. Most are able to mimic a very specific human function. Nevertheless, because of many challenging questions, the possibility of building an autonomous robot that uses the same principles as the human brain for decision making and surviving in an unfriendly world remain unanswered. Hence now may be the time to try a different approach, to formulate the dynamical principles that are common in both animals and intelligent robots. These same principles may serve as the basis for the creation of a brainmachine interface that can lead to a new generation of neural prosthetics and hybrid "brain-computer" systems. Contributing to the formulation of those underlying dynamical principles is the primary goal of this special issue of Biological Cybernetics.

The special issue reflects the bidirectional interaction between neurobiology on the one hand and engineering on the other. It addresses two main questions: First, what can neuroscience contribute to neuroprosthetics and intelligent robot design? Second, how can robots and brain-machine interfaces help us to understand better the principles of brain function? The improvement in yield, stability, and longevity of recordings, and new

A. J. Ijspeert

School of Computer and Communication Sciences EPFL,

Ecole Polytechnique Fédérale de Lausanne,

EPFL-IC-ISIM-GRIJ, Station 14,

Ch 1015 Lausanne, Switzerland

e-mail: auke@ijspeert.epfl.ch imaging techniques, combined with new dataprocessing methods, have allowed neurophysiologists to describe brain activities in terms of the dynamics of spatiotemporal patterns. It is not completely clear, however, how to interpret the corresponding information and how to use it for the control of artificial motor systems such as prosthetic devices or robots. Despite this, the success of a brain-motor-interface approach leads to a better understanding of brain motor control and brain dynamics.

Building artificial connections between the brain and the world is an extremely challenging problem for neuroscientists, physicists, mathematicians, and engineers that requires answers to several key questions:

1. How do the different time scales of activities in the brain that are involved in behavioral organization coordinate with each other?

2. How can a very complex dynamical brain, with a huge amount of neuronal activity, generate a behavioral command?

3. What mechanisms are responsible for dimension reduction and what are the cooperative brain variables that control motor systems?

4. How do sensory signals support stable, robust, and reproducible behaviors? We think that an understanding of the principles of dimension reduction, from high-dimensional brain dynamics to relatively low-dimensional behavioral activities, is one of the most important questions to answer at present. The major challenges to date for addressing this question have been to identify the patterns of cooperative dynamics and to find the minimal number of variables that in fact control behavioral activity. 
Animals and intelligent robots are integrated into the surrounding dynamical world through sensory and motor systems. Such interactions must be sensitive to the most important signals in the environment, but at the same time they must be robust against noninformative perturbations. Animals are able to solve this fundamental contradiction between sensitivity and robustness. Of course, a well-coordinated behavior is the result of informational feedback between motion and sensory systems, and it is reasonable to hypothesize that such "feedforward-feedback" interactions of the motor activity with the generation and representation of the behavioral information in the brain result in effective brain dynamics with a relatively low-dimensional output.

When we model the nervous system of an animal or the biomimetic "brain" of a robot as a dynamical system, three conditions must hold: (a) the model must be robust against noise and at the same time sensitive to incoming information from the environment, (b) the behavior of the model must be reproducible but at the same time flexible and adaptive, and (c) the model must be organized hierarchically in time, i.e., each behavioral event is the initial condition for the next window of time. We just mentioned the most commonly discussed principles, many of which are mutually contradictory (like robustness and sensitivity), but at the recent EPFLLATSIS 2006 Symposium, which led to this special issue, participants demonstrated considerable optimism based on the following three arguments.

First, neural systems are the result of evolution, and thus their complexity is not arbitrary but follows some universal rules. One such rule is that the organization of the central nervous system is hierarchical and based on neural modules (Rabinovich et al.).

Second, it is important to note that many modules are organized in a very similar manner across different species. Such units can be small, like central pattern generators (Selverston and Ayers; Vogelstein et al.), or much more complex, like sensory systems. In particular, the structure of one of the oldest sensory systems, the olfactory system, is more or less the same in invertebrates and vertebrates and can be described by similar dynamical models (Rabinovich et al.). Similarly, the same modules might be reused in several parts of the brain for different functions, as illustrated by the concept of neural fields (Sauser and Billard).

Third, the possibility of considering the nervous system as an ensemble of interconnected units is a result of the high level of autonomy of its subsystems (Selverston and Ayers; Vogelstein et al.; Kuniyoshi and Sangawa; Frigon and Rossignol). The level of autonomy depends on the degree of self-regulation in neural modules at each level of the nervous system, including individual neurons. This may be a key principle determining hierarchical neural network dynamics. The implementation of these ideas in real-world systems is discussed using a cybernetic hand (Carrozza et al.), a biped humanoid robot (Vogelstein et al.), and oscillatory systems for engineering applications (Buchli et al.).

This special issue is a followup of the EPFL-LATSIS 2006 Symposium that took place in Lausanne (Switzerland) on 8-10 March 2006 and addressed exactly the same topics. The conference brought together neuroscientists, physicists, mathematicians, and engineers interested in understanding the dynamical properties of the nervous system and in taking inspiration from these properties for the design of prosthetic and robotic devices. We hope that the December 2006 issue will be as inspiring to the reader as the meeting was to us.

\section{Jonas Buchli \\ Auke Jan IJspeert \\ Mikhail Rabinovich \\ Allen Selverston}

